\title{
Fluvoxamine for COVID-19 ICU patients?
}

\author{
Vladimir Trkulja ${ }^{1}$ \\ ${ }^{1}$ Department of Pharmacology, School of Medicine, University of Zagreb
}

November 18, 2021

\section{Fluvoxamine for COVID-19 ICU patients?}

Vladimir Trkulja

Department of Pharmacology

Zagreb University School of Medicine

Šalata 11, Zagreb, Croati

vladimir.trkulja@mef.hr

Number of words: 800

Number of figures/tables: 1

To the Editor,

I read with interest a recently BJCP-accepted manuscript on the use of fluvoxamine in COVID-19 patients who needed admission to an intensive care unit (ICU) ${ }^{1}$. It was instructive to read about the pre-existing clinical experience and about possible mechanisms of presumed benefits of fluvoxamine in COVID-19. However, attention needs to be drawn to the suggested effect of fluvoxamine quantified as a $40 \%$ reduction in instantaneous risk of death. The authors report ${ }^{1}$ on a cohort $(\mathrm{n}=51)$ of patients who, upon ICU admission, were treated with oral fluvoxamine added to the standard of care (SoC) $(3 \times 100 \mathrm{mg} /$ day over 15 days, then $2 \times 50 \mathrm{mg}$ /day over 7 days), and who were compared to a cohort $(\mathrm{n}=51)$ of SoC-only patients. The two cohorts were said to be matched ${ }^{1}$. Based on reported data ${ }^{1}$, it appears that the patients were matched exactly in respect to gender and COVID-19 vaccination status, and, seemingly, on a rather narrow age-caliper, but the matching method was not reported ${ }^{1}$; not reported was also a measure of matching adequacy - standardized difference $(d)$, a preferred method of balance assessment (adequate if $d<0.1$ ) since independent of the sample size ${ }^{2}$. Based on the reported data ${ }^{1}$, for example, the fluvoxamine $-S o C d$ regarding body mass index was -0.30 ( -0.31 in women and -0.29 in men); also, $d=-0.122$ regarding history of diabetes, $d=-0.350$ regarding history of treated hypertension, $d=-0.11$ regarding on-admission APACHE score - all suggesting a considerable imbalance between the two cohorts (lower values in the fluvoxamine cohort). The authors provide Kaplan-Meier curves of time-to-death (or ICU discharge) but without the numbers at risk ${ }^{1}$. Still, data could be read from the graphs and curves reconstructed (Figure 1A):(i) the first marked difference between the treated and controls occurs during the first 7 days of observation -3 patients died and 3 were censored in the former, and 11 died and 4 were censored the latter cohort (Figure 1A). This difference in deaths (3 vs. 11) did not change over the entire later period since the overall difference in the number of deaths was $9(30 / 51$ in treated vs. 39/51 in controls). This would indicate a very rapid-onset (and subsequently "lost") effect of fluvoxamine, which does not seem pharmacologically plausible. The assumed fluvoxamine mechanisms $^{1}$ are not of the immediate-onset type; with a $3 \times 100 \mathrm{mg}$ /day dosing, elimination half-life is likely to extend well beyond 30 hours, hence steady-state would be achieved only after 7-10 days ${ }^{3}$. Combined with the baseline imbalance between groups, this indicates that the initial separation of the two curves - more 
or less preserved throughout the entire subsequent period - was likely not attributable to fluvoxamine; (ii) after day 21 , and particularly after day 28 , the numbers at risk were very low, and after day 35 there were no further events (Figure 1A), hence accounting for the entire curve is likely misleading ${ }^{4}$; (iii) although the curves do not cross (Figure 1A), they indicate a possibility that hazard ratio varied over time. Hazard ratio as generated in a Cox proportional hazard model (as done by the authors) is an average of values that can change over time $\mathrm{e}^{5}$; it is also inherently prone to selection bias and, even in absence of confounding its interpretation is not straightforward ${ }^{5}$. This holds for randomized and particularly for non-randomized settings ${ }^{5}$. Reconstructed data depicted in Figure 1A were used to fit a complementary log-log model for continuous time process taking into account the first 35 days (no events after that point): the method treats time as a continuous but more "coarsely" measured variable, in intervals of identical length (in this case 7-day intervals, i.e., weeks); based on assumption of constant hazard within the interval, the method provides period-specific (for weeks 1-5) hazard ratios ${ }^{6}$, which is likely a preferable option ${ }^{5}$. Figure $1 \mathrm{~B}$ depicts estimated probabilities of death and HRs: it is only during week 1 that the hazard appeared lower in treated - a period during which, as elaborated, fluvoxamine most likely had no effect. Finally, authors fitted a multivariable Cox model ${ }^{1}$ to substantiate the fluvoxamine effect. With a total of 15 independents in a study with 102 subjects, the model was likely overfitted and susceptible to bias arising from over(unnecessary)-adjustments ${ }^{7}$. But more importantly, it included adjustment for renal replacement therapy (RRT), which was actually one of the outcomes. Inadequacy of adjustments for post-exposure outcomes as if they were baseline covariates has been extensively elaborated ${ }^{8}$ and almost inevitably results in a considerable bias, regardless of whether the respective variable was actually a mediator or a collider ${ }^{8}$. Such adjustments require implementation of marginal structural models or some of the $g$-estimation methods ${ }^{9}$.

Overall, the reported difference between the two cohorts of patients is more likely bias arising from design and analysis than evidence supporting a causal effect of fluvoxamine.

\section{References}

1. Čalušić M, Marčec R, Lukša L et al. Safety and efficacy of fluvoxamine in COVID-19 ICU patients: an open label, prospective cohort trial with matched controls. Br J Clin Pharmacol . 2021; doi: $10.1111 /$ bcp. 15126 .

2. Stuart EA. Matching methods for causal inference: a review and a look forward. Stat Sci . 2010; $25(1): 1-21$.

3. Hiemke C, Hartter S. Pharmacokinetics of selective serotonin reuptake inhibitors. Pharmacol Ther . 2000; 85 (1):11-28.

4. Machin D, Cheung YB, Parmar MKB, eds. Survival analysis: a practical approach . $2^{\text {nd }}$ ed. Chichester, West Sussex: John Wiley \& Sons Ltd; 2006. p.38.

5. Hernan MA. The hazards of hazard ratios. Epidemiology 2010; 21(1):13-15.

6. Prentice RL, Gloecker LA. Regression analysis of grouped survival data with application to breast cancer data. Biometrics 1978; 34(1):57-67.

7. Schisterman EF, Core SF, Platt RW. Overadjustment bias and unnecessary adjustment in epidemiological studies. Epidemiology 2009; 20(4):488-495.

8. Greenland S. Quantifying biases in causal models: classical confounding vs collider-stratification bias. Epidemiology 2003; 14(4):300-306.

9. Hernan MA, Robins JM, eds. Causal inference: What if . $1^{\text {st }}$ ed. CRC Press LLC; 2019.

Figure 1. Summary of re-analysis of survival data published in ref. 1. A . Reconstructed curves of Kaplan-Meier product-limit estimates. Data ${ }^{1}$ were read using a digitizing software, and were re-analyzed and curves were drawn using JMP 13 software (SAS Institute Inc., Cary, NC). Upward oriented ticks indicate censorings, downward oriented ticks indicate failures. ICU - intensive care unit. B . Estimated probabilities of death during weeks 1 to 5 by treatment (Fluvox - fluvoxamine) and period-specific hazard ratios (HR) with confidence intervals. A complementary log-log model was fitted to reconstituted data using SAS 9.4 for Windows (SAS Inc., Cary, NC).

\section{Hosted file}


Trkulja LET-01276-21 Fig01.docx available at https://authorea.com/users/437234/articles/ 545956-fluvoxamine-for-covid-19-icu-patients 